\title{
The Role of Residual Symmetries in Dark Matter Stability and the Neutrino Nature
}

\author{
Cesar Bonilla, ${ }^{1}$ Eduardo Peinado, ${ }^{2}$ and Rahul Srivastava ${ }^{3}$ \\ ${ }^{1}$ Physik-Department T30d, Technische Universität München. \\ James-Franck-Strasse, 85748 Garching, Germany \\ 2 Instituto de Física, Universidad Nacional Autónoma de México, A.P. 20-364, Ciudad de México 01000, México. \\ ${ }^{3}$ AHEP Group, Institut de Física Corpuscular - CSIC-Universitat de València, Parc Científic de Paterna. \\ C/ Catedrático José Beltrán, 2 E-46980 Paterna (Valencia) - SPAIN
}

\begin{abstract}
We consider the class of models where Dirac neutrino masses at one loop and the dark matter stability can be obtained using only the global $U(1)_{B-L}$ symmetry already present in Standard Model. We discuss how the residual $\mathcal{Z}_{n}$ subgroup, left unbroken after the breaking of $U(1)_{B-L}$, dictates the neutrino nature, namely if they are Dirac or Majorana particles, as well as determines the stability of the dark matter candidate in such models. In particular, we show that without the correct breaking of $U(1)_{B-L}$ to an appropriate residual $\mathcal{Z}_{n}$ symmetry, the Dirac nature of neutrinos and/or dark matter stability might be lost. For completeness, we also provide some examples where the dark matter stability is accidental or lost completely. Finally, we discuss one example model where the Dirac neutrinos with naturally small one loop masses as well as dark matter stability, are both protected by the same residual $\mathcal{Z}_{6}$ subgroup, without need for adding any new explicit or accidental symmetries beyond the Standard Model symmetries.
\end{abstract}

Keywords: Dirac Neutrinos, Loop Mass Mechanism, Matter, Residual Symmetry

DOI: $10.31526 /$ lhep.1.2019.124

\section{INTRODUCTION}

The Standard Model is the best description we have so far to explain all the observed fundamental particles and their interactions, namely the strong and electroweak phenomena. However, the Standard Model predicts that neutrinos are massless particles and lacks a candidate to account for the dark matter relic abundance in the Universe [1].

Neutrino oscillation experiments indicate that at most one active neutrino can be massless $[2,3,4,5]$ but there is no experimental hint pointing towards the exact mechanism to generate mass for neutrinos. In this regard, the most popular approach to alleviate this Standard Model shortcoming is to assume that neutrinos are Majorana in nature and invoke the so-called Majorana seesaw mechanisms $[6,7,8,9,10,11]$. In fact, independent of the Dirac/Majorana nature of neutrinos and the details of mass generation mechanism, neutrino mass generation always requires the existence of new particles and/or symmetries $[12,13]$.

From the theoretical perspective, it is very tempting to think that one of the new fundamental fields added to the Standard Model to generate the neutrino mass might be a potential dark matter candidate. The connection between dark matter and the neutrino mass naturally arises when the mass generation mechanism is at the loop level. The simplest realization of this idea is achieved within the scotogenic model [14]. Besides the Standard Model particle content, in this scenario the neutrino mass is generated at the one-loop level by assuming the existence of $\mathrm{SU}(3)_{\mathrm{C}} \otimes \mathrm{SU}(2)_{\mathrm{L}} \otimes \mathrm{U}(1)_{\mathrm{Y}}$ gauge singlet fermions and an extra $S U(2)_{L}$ doublet scalar with vanishing vacuum expectation value (vev). All these new particles carry an odd charge under a global $\mathcal{Z}_{2}$-symmetry. This additional $\mathcal{Z}_{2}$ symmetry remains unbroken after the electroweak symmetry breaking (EWSB). It stabilizes the dark matter candidate and forbids the appearance of Majorana mass terms for the neutrinos at tree-level.

Following the scotogenic idea, here we will consider a class of models [15] where ${ }^{1}$

I. Neutrinos are Dirac in nature.

II. Neutrino mass is generated at one loop level.

III. In scotogenic spirit, the intermediate particles in the loop belong to a "dark sector" with the lightest particle among them being a good candidate for stable dark matter.

Typically, one needs to invoke several additional symmetries to achieve this. However, as has been shown in [15], for certain models this can be achieve only through the global $U(1)_{B-L}$ of the Standard Model ${ }^{2}$. In this setup the global $U(1)_{B-L}$ symmetry of Standard Model is broken down to its residual $\mathcal{Z}_{n}$; $n \in \mathbb{Z}^{+}$subgroup. Since we require neutrinos to be Dirac particles, this residual symmetry should be such that it can protect the Dirac nature of neutrinos as well as stabilize the dark matter candidate [15]. This can be successfully done only if the breaking $U(1)_{B-L} \rightarrow \mathcal{Z}_{n}$ is achieved in a correct manner such that an appropriate $\mathcal{Z}_{n}$ subgroup is left unbroken. Not all $\mathcal{Z}_{n}$ subgroups of $U(1)_{B-L}$ can do this job. The criterion for such an appropriate residual $\mathcal{Z}_{n}$ are also listed in $[15,16]$. In this paper, we aim to construct a class of models and analyze them in details, in order to highlight the crucial role that the residual $\mathcal{Z}_{n}$ plays in protecting the Dirac nature of neutrinos as well as in dark matter stability.

\footnotetext{
${ }^{1}$ For analogous framework for Majorana neutrinos see [16].

${ }^{2}$ The idea of obtaining dark matter stability associated to the breaking of $U(1)_{B-L}$ has been explored in $[17,18,19,20,21,22,23,24,13,25,26,15,27,16]$ For recent works aimed at obtaining scotogenic stability from extended gauge symmetries see $[28,29]$.
} 
This work is organized as follows: in the next section, we describe how the residual symmetry is connected to the neutrino nature. In section 3 , the list of possible realizations to generate the neutrino mass dynamically is given. In order to highlight the important role of the residual symmetry in ensuring the dark matter stability, we provide several explicit examples in Section 4, where, due to lack of an appropriate residual symmetry, the dark matter stability is lost. For completeness, we also give examples of the cases where an additional accidental symmetry might be present in the model leading to dark matter stability. In Section 5, we present a model where the residual $\mathcal{Z}_{6}$ symmetry protects both the stability of the dark matter candidate and Dirac nature of neutrinos. We conclude after that.

\section{RESIDUAL SYMMETRIES AND THE NEU- TRINO NATURE}

Typically in high energy physics models, when a symmetry $G$ is broken down either spontaneously or explicitly, a subgroup of it remains unbroken. This unbroken subgroup $G_{\text {res }}$ is referred to as the residual subgroup. It is this residual subgroup $G_{\text {res }}$ and not the full group $G$, which dictates the dynamics of a given theory at energy and temperatures below the scale of $G$ breaking. The most well known example of this is provided by Standard Model itself. In Standard Model, the gauge group $\mathrm{SU}(3)_{\mathrm{C}} \otimes \mathrm{SU}(2)_{\mathrm{L}} \otimes \mathrm{U}(1)_{\mathrm{Y}}$ is spontaneously broken down to a residual subgroup $G_{\text {res }} \equiv \mathrm{SU}(3)_{\mathrm{c}} \otimes \mathrm{U}(1)_{\text {em }}$ by the vev of the Higgs. Below the electroweak breaking scale, the dynamics of the Standard Model is dictated by the unbroken residual subgroup $\mathrm{SU}(3)_{\mathrm{c}} \otimes \mathrm{U}(1)_{\mathrm{em}}$. Apart from this well known example, residual symmetries occur in almost all beyond Standard Model (BSM) extensions, with $\mathrm{SU}(3)_{\mathrm{C}} \otimes \mathrm{SU}(2)_{\mathrm{L}} \otimes \mathrm{U}(1)_{\mathrm{Y}}$ itself being a residual subgroup in most of the BSM gauge extensions. Coming back to neutrinos, the residual symmetries play an important role in determining the Dirac/Majorana nature of neutrinos and might also be responsible for dark matter stability. We first highlight their role in determining the nature of neutrinos before discussing their role in dark matter stability.

To see the important role played by residual symmetries in determining the nature of neutrinos, recall that apart from $\mathrm{SU}(3)_{\mathrm{C}} \otimes \mathrm{SU}(2)_{\mathrm{L}} \otimes \mathrm{U}(1)_{\mathrm{Y}}$ gauge symmetries, the Standard Model also has two other symmetries namely Lepton number $U(1)_{L}$ and Baryon number $U(1)_{B}$ symmetries. The combination $U(1)_{B-L}$ can be rendered anomaly free if we extend the Standard Model particle content by adding three right handed neutrinos $v_{R_{i}} ; i=1,2,3$ either carrying vector charges $(-1,-1,-1)$ or chiral charges $(-4,-4,5)$ under $U(1)_{B-L}$ symmetry $[30,31,32,33]$. Given that the Standard Model lepton doublets $L_{i} ; i=1,2,3$ carry $U(1)_{B-L}$ charge, the Dirac or Majorana nature of neutrinos depends crucially on the residual symmetry $G_{\text {res }}$ appearing after the breaking of the $U(1)_{B-L}$ symmetry. Since $U(1)_{B-L}$ is an Abelian continuous group, it only has discrete Abelian subgroups, namely $G_{\text {res }} \equiv \mathcal{Z}_{m} ; m \in \mathbb{Z}^{+}$. Depending on the nature of the residual $\mathcal{Z}_{m}$ subgroup left unbroken, one can classify the Dirac or Majorana nature of neutrinos, depending on how Standard Model lepton doublets $L_{i}$; $i=1,2,3$ transform under the residual subgroup ${ }^{3}$. If $U(1)_{B-L}$

${ }^{3}$ If there are new, as of yet unknown, conserved symmetries present in nature, the classification presented here should be generalized to include them. Such a generalization is straightforward. remains unbroken then neutrinos will be Dirac particles as the Majorana mass term for neutrinos is forbidden by $U(1)_{B-L}$ symmetry. In case $U(1)_{B-L} \rightarrow \mathcal{Z}_{m}$ we have [34].

$$
\begin{aligned}
U(1)_{B-L} & \rightarrow \mathcal{Z}_{m} \equiv \mathcal{Z}_{2 n+1} \text { with } n \in \mathbb{Z}^{+} \\
& \Rightarrow \text { Neutrinos are Dirac particles } \\
U(1)_{B-L} & \rightarrow \mathcal{Z}_{m} \equiv \mathcal{Z}_{2 n} \text { with } n \in \mathbb{Z}^{+} \\
& \Rightarrow \text { Neutrinos can be Dirac or Majorana }
\end{aligned}
$$

Now, if the $U(1)_{B-L}$ is broken to a $\mathcal{Z}_{2 n}$ subgroup, then one can make a further classification depending on how the $L_{i}$ transform,

$L_{i} \begin{cases}\nsim \omega^{n} & \text { under } \mathcal{Z}_{2 n} \quad \Rightarrow \text { Dirac Neutrinos } \\ \sim \omega^{n} \text { under } \mathcal{Z}_{2 n} & \Rightarrow \text { Majorana Neutrinos }\end{cases}$

where $\omega=e^{2 \pi I / 2 n} ; \omega^{2 n}=1$ is the $2 n$-th root of unity.

In order to understand last statement better, one can take, for instance, the dimension-5 Weinberg operator [35] for Majorana neutrino mass $\bar{L}^{c} L H H$ (where $L$ and $H$ are $S U(2)_{L}$ lepton and scalar Higgs doublets, respectively) which basically breaks lepton number leaving a residual $\mathcal{Z}_{2}$ symmetry unbroken. Neutrinos will be Majorana in this case as it satisfies the criterion for Majorana neutrinos, listed in (2). As an example of a ultra violet (UV) completion, one can take the type-I seesaw mechanism, where three RH-neutrinos are added to the Standard Model, transforming as $\left(v_{R_{i}}\right) \sim(-1)$ (with $i=1,2,3$ ) under the global $U(1)_{B-L}$ symmetry. In order to generate the Majorana mass term for neutrinos, a lepton number breaking term leading to $U(1)_{B-L} \rightarrow \mathcal{Z}_{2}$ has to be added to the Lagrangian. Hence,

$\mathcal{L}_{\succeq}=y^{v} \bar{L} \tilde{H} v_{R}+M_{R} \bar{v}_{R}^{c} v_{R}+$ h.c.

where we have omitted the flavor indices for convenience. In this case the mass term $M_{R} \bar{v}_{R}^{c} v_{R}$ softly breaks the $U(1)_{B-L} \rightarrow$ $\mathcal{Z}_{2}$ and hence neutrinos are Majorana in nature. On the other hand, if a dynamical origin of the neutrino mass is demanded, one has to further assume the existence of a scalar singlet $\sigma$ with non-trivial charge under $U(1)_{B-L}$, i.e. $\sigma \sim \pm 2$ should be chosen. As consequence, the vev of this scalar $\langle\sigma\rangle$ will break $U(1)_{B-L} \rightarrow \mathcal{Z}_{2}$ ultimately generating the Majorana mass for neutrinos $[8,10]$.

To obtain Dirac neutrinos one must break $U(1)_{B-L} \rightarrow \mathcal{Z}_{n}$ with $n \geq 3$ as the residual subgroup $\mathcal{Z}_{2}$ always leads to Majorana neutrinos in accordance with (2). While traditionally mass models for Majorana neutrinos have garnered lot of attention, in recent years, Dirac neutrino mass models are enjoying a resurgence of sorts with various seesaw [31, 32, $33,36,20,21,37,23,38,39,40,41,42,43]$ and loops models $[44,45,46,22,47,24,48,49,25,50,15,27,51,28,52,53]$ considered in literature ${ }^{4}$. The operator based classification of such models at dimension-4 [58], dimension-5 [59, 13] and dimension-6 [60, 61] have also been considered.

From now on, the focus is to explain the smallness of the neutrino mass when neutrinos are Dirac particles in nature. That is, there should be a dynamical mass mechanism behind the Dirac neutrino mass generation. In order to have a natural explanation for smallness of Dirac neutrino masses, it is desirable to forbid the tree-level neutrino Yukawa coupling

\footnotetext{
${ }^{4}$ For Dirac neutrino models without mass mechanism see $[54,55,56,57,34]$.
} 
$\mathcal{L}_{v} \supset y^{v} \bar{L} \tilde{H} v_{R}$. This coupling can be forbidden in many ways, for example by imposing flavor symmetries [21,23] or even by a simple $\mathcal{Z}_{2}$ symmetry [20]. It can be automatically forbidden by the $U(1)_{B-L}$ symmetry itself, if we use the chiral solution of $\left(v_{R_{a}}, v_{R_{3}}\right) \sim(-4,5)$ (with $\left.a=1,2\right)$, as was shown for the first time in $[31,32,33]$. The operator that generates the neutrino mass will then appear at higher dimensional level. For this to happen, a singlet scalar field $\chi \sim 3$ under the $U(1)_{B-L}$ has to be added in order to generate the mass of at least two neutrinos through a dimension 5 operator. The dimension 5 operator then is given by

$\mathcal{L}_{v} \supset \frac{y^{v}}{\Lambda} \bar{L} \tilde{H} \chi v_{R_{a}}$

where $\Lambda$ is the scale of UV completion. The mass of the third neutrino can also be generated by either the dimension- 6 term

$\mathcal{L}_{v} \supset \frac{y^{v}}{\Lambda^{2}} \bar{L} \tilde{H} \chi^{*} \chi^{*} v_{R_{3}}$

or by adding a new singlet scalar $\chi_{6} \sim 6$ under $U(1)_{B-L}$ such that the dimension- 5 term

$\mathcal{L}_{v} \supset \frac{y^{v}}{\Lambda} \bar{L} \tilde{H} \chi_{6}^{*} v_{R_{3}}$

is also allowed by the $U(1)_{B-L}$ symmetry.

The UV completion of such operators is model dependent, as we will discuss in Section 3. Since the field $\chi \sim 3$ under $U(1)_{B-L}$, its vev will break $U(1)_{B-L} \rightarrow \mathcal{Z}_{3 m} ; m \in \mathbb{Z}^{+}$. The exact residual $\mathcal{Z}_{3 m}$ subgroup will depend on the details on the UV completion, a fact we discuss in further details in Section 4. Also, note that, since the $U(1)_{B-L}$ charge of $\chi_{6} \sim 6$ is a multiple of the charge of $\chi \sim 3$, the addition of $\chi_{6}$ in a given UV complete model will not change the nature of the residual $\mathcal{Z}_{3 m}$ symmetry. Before moving on, let us briefly see the various possible ways the operator in (4) can be UV completed at one-loop.

\section{ONE-LOOP TOPOLOGIES OF THE OPERATOR $\bar{L} \tilde{H} \chi v_{R_{A}}$}

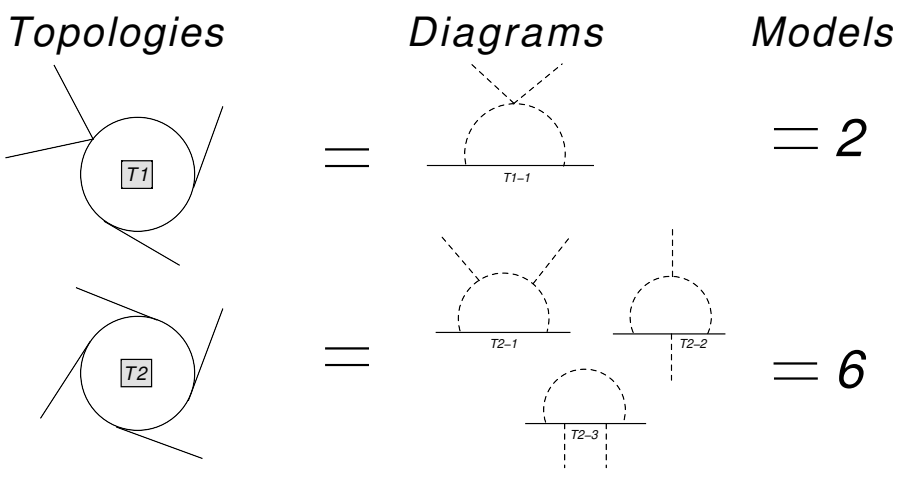

Figure 1: One loop topologies with four external lines.

In this section, we provide the topologies and all possible oneloop diagrams for the dimension-5 operator $\bar{L} \tilde{H} \chi v_{R_{a}}$, that generate Dirac neutrino masses. We have associated each topology to graphs or Feynman diagrams without taking into account their Lorentz nature. We understand as diagrams when the fermion and scalar lines are specified. We consider the case where the neutrino masses arise after spontaneous breaking of the $\mathrm{SU}(3)_{C} \otimes S U(2)_{L} \otimes U(1)_{Y}$ and $U(1)_{B-L}$ symmetry. Under this requirement the lowest order comes from topologies at dimension-5 [27], as shown in Figure 1. This implies that in the diagrams corresponding to topologies T1 and T2, two external legs are fermions (continuous lines) and the others are scalars (dashed lines). One of these scalars corresponds to the Standard Model Higgs doublet which is responsible for the EWSB and the other one, the scalar singlet $\chi$, is assigned to break $U(1)_{B-L}$ symmetry.

It turns out that within the T1 topology, there is only one possible diagram called T1-1 in Figure 1. There are two possible realizations of such a diagram depending on whether the internal fermions are $S U(2)_{L}$ doublets or singlets ${ }^{5}$. This is in contrast to the classification provided in [27]. For the T2 topology, three different dimension-5 diagrams (T2-1, T2-2, T2-3) can be drawn. In this case, the different models that can be constructed also depend on the $S U(2)_{L}$ representations of the internal fields. It is important to mention that all these diagrams are genuine in the sense that there is no lower order contribution to neutrino mass. That is, there is no tree-level contribution to the neutrino mass generation out of the matter content for these cases. Note that within a model where the neutrino mass is generated through a T2-1 diagram it can have a T1-1 diagram when the scalar on top is integrated out. Regardless this, T2-1 remains being genuine under our previous definition. Having considered all possible topologies and diagrams for one loop realization of the operator $\bar{L} \tilde{H} \chi v_{R_{a}}$ we now turn to the scenarios where the intermediate particles can belong to a dark sector with the lightest one being a potential dark matter candidate. The dark matter stability in such scenarios again depends crucially on the residual $\mathcal{Z}_{n}$ symmetry which we discuss with explicit examples in next section.

\section{RESIDUAL SYMMETRY AND DARK MATTER STABILITY}

We already saw the crucial role the residual symmetries play in determining the Dirac or Majorana nature of neutrinos. They play an equally important role in determining the stability of the potential dark matter candidate in a given model. In this section, we elucidate this role further by taking the one loop completion of the dimension-5 operator $\bar{L}_{i} \tilde{H} \chi v_{R_{a}} ; i=1,2,3$ and $a=1,2$ of previous section and scrutinizing it in more details.

As discussed in details in Section 2, taking the anomaly free solution with $v_{R}=(-4,-4,5)$ under $U(1)_{B-L}$ has the advantage that the tree level Yukawa term is automatically forbidden. However, the dimension-5 operator $\bar{L} \tilde{H} \chi v_{R_{a}}$ is allowed by $U(1)_{B-L}$ and paves way for generating small Dirac masses at one loop level as shown in $[15,27]$. Once the scalar $\chi$ carrying 3 units of $U(1)_{B-L}$ charge gets vev, the $U(1)_{B-L}$ symmetry is broken to a residual $\mathcal{Z}_{n}$ subgroup. In such a setup, without adding any extra symmetry, the intermediate particles running in the loop can in principle be arranged to belong to the "dark

\footnotetext{
${ }^{5}$ In this work, we restrict to only colorless $S U(2)_{L}$ singlet and doublet representations. Models with intermediate fields carrying non-trivial color, exotic hypercharge or having higher $S U(2)_{L}$ representations are also possible but will not be considered here.
} 


\begin{tabular}{|c|c|c|c|c|}
\hline & Fields & $S U(2)_{L} \otimes U(1)_{Y}$ & $U(1)_{B-L}$ & $\mathcal{Z}_{3}$ \\
\hline 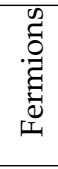 & $\begin{array}{c}L_{i} \\
v_{R_{a}} \\
v_{R_{3}} \\
N_{L(R)} \\
\end{array}$ & $\begin{array}{c}(2,-1 / 2) \\
(\mathbf{1}, 0) \\
(\mathbf{1}, 0) \\
(\mathbf{1}, 0) \\
\end{array}$ & $\begin{array}{c}-1 \\
-4 \\
5 \\
9\end{array}$ & $\begin{array}{l}\omega^{2} \\
\omega^{2} \\
\omega^{2} \\
\omega^{q}\end{array}$ \\
\hline$\frac{\infty}{\overparen{J}}$ & $\begin{array}{l}H \\
\chi \\
\eta \\
\xi\end{array}$ & $\begin{array}{c}(2,1 / 2) \\
(1,0) \\
(2,1 / 2) \\
(1,0)\end{array}$ & $\begin{array}{c}0 \\
3 \\
1-|q| \\
4-|q|\end{array}$ & $\begin{array}{c}\omega^{0} \\
\omega^{0} \\
\omega^{1-|q|} \\
\omega^{1-|q|}\end{array}$ \\
\hline
\end{tabular}

Table 1: Matter content and charge assignments for realizing T1-1. For all integer values of $B-L$ charge $q$, the $U(1)_{B-L} \rightarrow \mathcal{Z}_{3}$. The residual $\mathcal{Z}_{3}$ charges are shown in last column where $\omega=e^{2 \pi I / 3} ; \omega^{3}=1$ is the cube root of unity.

sector" with the lightest of them being potentially stable à la scotogenic mechanism [14]. However, whether such a potential dark matter particle will be stable or not is dictated by the residual $\mathcal{Z}_{n}$ subgroup and not by the $U(1)_{B-L}$ symmetry $[15,16]$. In this section, we demonstrate this fact explicitly by taking the model realizations of the T1 topology of Section 3. A similar analysis can also be done for all other diagrams belonging to the T2 topology.

The first thing to notice is that when $U(1)_{B-L} \rightarrow \mathcal{Z}_{n}$, the type of residual $\mathcal{Z}_{n}$ subgroup that is left unbroken depends on the vev of the $U(1)_{B-L}$ charge carrying scalar as well as on the details of charges of the intermediate fields required for UV completion. For the operator $\bar{L}_{i} \tilde{H} \chi v_{R_{a}} ; i=1,2,3$ and $a=1,2$, since $\chi \sim 3$ under $B-L$, the residual $\mathcal{Z}_{n}$ subgroup can only be a $\mathcal{Z}_{3 m} ; m \in \mathbb{Z}^{+}$group. However, exactly which $\mathcal{Z}_{3 m}$ is left unbroken after the breaking of the $U(1)_{B-L}$ symmetry, is dependent on the details of a given model and in particular to the lowest $U(1)_{B-L}$ charge in the model. Now we further illustrate this fact with explicit examples.

To see the crucial role of residual symmetry in dark matter stability, lets consider the model realizations of the T1-1 diagram of Figure 1. The diagram can be UV completed by adding new $\mathrm{SU}(3)_{\mathrm{C}} \otimes \mathrm{SU}(2)_{\mathrm{L}} \otimes \mathrm{U}(1)_{Y}$ singlet fermions $N_{L}, N_{R}$ and scalar $\xi$ along with the inert $S U(2)_{L}$ doublet scalar $\eta$. All these fields are charged under $U(1)_{B-L}$ symmetry with their charges as listed in Table 1.

As shown in Table 1, the one-loop neutrino mass can be generated for all values of the $U(1)_{B-L}$ charge $q$. However, the residual symmetry in all cases need not be same. To begin with, lets consider the cases where $q \in \mathbb{Z}$, i.e., $q$ only takes integer values. In all such cases, the lowest $U(1)_{B-L}$ charge in the model is \pm 1 , the charge of the lepton doublets $L_{i}$. Since, $\chi \sim 3$ under $U(1)_{B-L}$, its vev will then break $U(1)_{B-L} \rightarrow \mathcal{Z}_{3}$. The residual $\mathcal{Z}_{3}$ symmetry, being an odd $\mathcal{Z}_{n}$ group, is enough to protect the Dirac nature of neutrinos in accordance with (1). The one loop Dirac neutrino mass realization for $q \in \mathbb{Z}^{-}$and $q \in \mathbb{Z}^{+}$are shown in Figure $2 \mathrm{a}$ and in Figure $2 \mathrm{~b}$, respectively. Notice that the $\mathrm{SU}(3)_{\mathrm{C}} \otimes \mathrm{SU}(2)_{\mathrm{L}} \otimes \mathrm{U}(1)_{Y}$ singlet fermions $N_{L}$ and $N_{R}$ simple switch roles for the $q \in \mathbb{Z}^{-}$and $q \in \mathbb{Z}^{+}$cases.

Notice that the neutral components of the intermediate particles in the loops of Figure 2 can in principle belong to the "dark sector" with the lightest of them being a good dark matter candidate à la scotogenic model. Although the residual $\mathcal{Z}_{3}$ symmetry is enough to protect the Dirac nature of neutrinos, it is not enough to protect the stability of a potential dark matter candidate. In fact, as has been argued in [15], any odd $\mathcal{Z}_{n}$
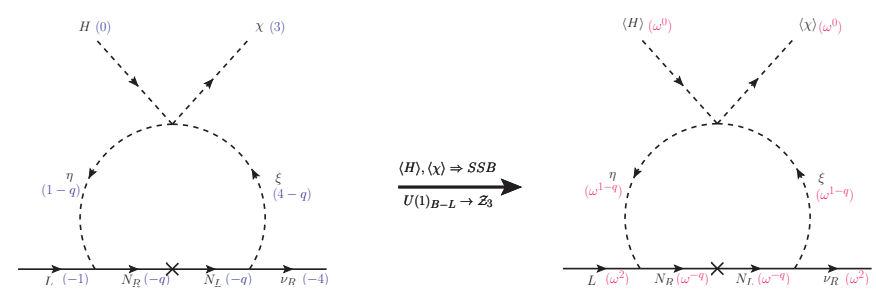

(a) For the case $q \in \mathbb{Z}^{-}$.
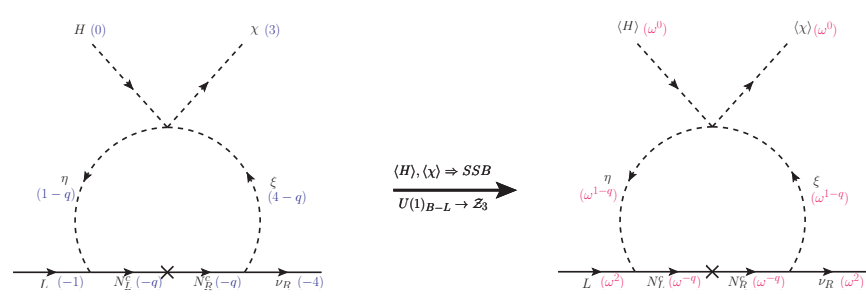

(b) For the case $q \in \mathbb{Z}^{+}$.

Figure 2: One loop neutrino mass generation diagrams highlighting the $U(1)_{B-L} \rightarrow \mathcal{Z}_{3}$ breaking pattern. The $B-L$ charges (left diagram) of the fields are in blue while the residual $\mathcal{Z}_{3}$ charges (right diagram) are in red. For $\mathcal{Z}_{3}$ symmetry $\omega=e^{2 \pi I / 3} ; \omega^{3}=1$ is the cube root of unity. Notice that the Standard Model gauge singlet fermions $N_{L}$ and $N_{R}$ have switched their roles in the two cases.

subgroup of $U(1)_{B-L}$, on its own cannot protect the dark matter stability in models employing scotogenic mechanism. The dark matter stability in all such models has to be achieved either by adding another explicit symmetry or by choosing "exotic" $U(1)_{B-L}$ charges for the intermediate fields such that an accidental symmetry also appears in the model. We now further elaborate on this by looking case by case at models with $q=0, \cdots \pm 6$. We start with looking at the cases where the dark matter stability is completely lost and then move on to the cases where a new accidental symmetry comes into play.

4.0.1. $q= \pm 1$ case

Let's start with the simplest case of $q= \pm 1$. For definiteness, we take the case of $q=-1$ but analogous discussion can be carried out for $q=+1$ case as well. The one loop neutrino mass generation diagram along with the residual $\mathcal{Z}_{3}$ charges is shown in Figure 3a.

However, for this case the Yukawa term $\bar{L} \tilde{H} N_{R}$ is also allowed by the symmetry. This term leads to mixing as well as decay of the $N_{R}$ fields as shown in Figure $3 b$. Since $N_{R}$ is a 


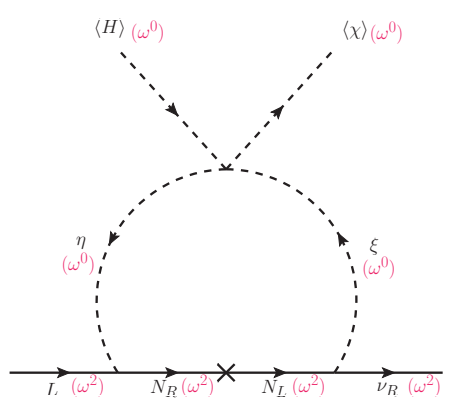

(a) The $q=-1$ case, raditative neutrino mass generation diagram along with residual $\mathcal{Z}_{3}$ charges. Analogous diagram can be drawn for $q=+1$ case, following Figure $2 b$.
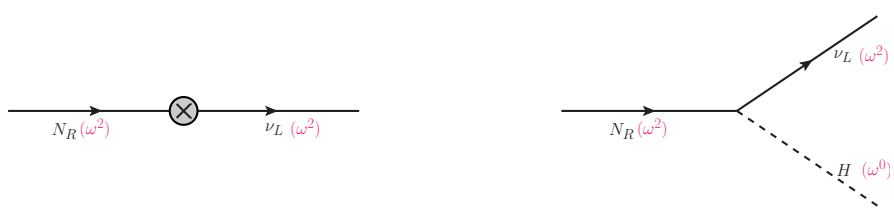

(b) The mixing and decay diagrams of dark sector particles, ultimately leading to the decay of potential dark matter candidate.

Figure 3: The raditative neutrino mass generation diagram and the mixing and decay diagrams of dark sector particle, for the $q=-1$ case. Anologous diagrams can also be drawn for $q=+1$ case with $N_{L}$ and $N_{R}$ switching their roles like in Figure 2a and Figure $2 \mathrm{~b}$

"dark sector" field, this coupling ultimately provides a channel for the dark matter decay, even if $N_{R}$ itself is the not the dark matter candidate. This is because just like in scotogenic model, here also, all intermediate fields can "decay" among each other. If one of them has a decay channel to Standard Model particles or to the new external fields $v_{R}, \chi$, then any potential dark matter candidate will ultimately decay by first going to real/virtual $N_{R}$ which will finally decay to $v_{L}$ and $H$ as shown in Figure $3 \mathrm{~b}$.

\subsection{2. $q= \pm 2$ case}

For the $q=-2$ case, the Feynman diagram leading to neutrino mass generation along with the residual $\mathcal{Z}_{3}$ charges of the fields is shown in Figure 4 a. As before, the case of $q=2$ can be analogously discussed with $N_{L}$ and $N_{R}$ fields switching their roles.

In this case, one can again write down a dimension- 4 term $\bar{N}_{R}^{c} v_{R_{3}} \chi^{*}$ which leads to the decay of $N_{R}$ field as shown in Figure $4 \mathrm{~b}$. As can be seen from Figure $4 \mathrm{~b}$, the residual $\mathcal{Z}_{3}$ symmetry does not forbid this decay. Now, since $\bar{N}_{R}^{c}$ is a dark sector particle, its decay channel ultimately provides a decay path for any potential dark matter candidate. Thus, the dark matter stability is again lost.

\subsection{3. $q= \pm 3$ case}

Turning to the $q= \pm 3$, the Feynman diagram for neutrino mass generation is shown in Figure 5a. The diagram in Figure 5a is drawn for the case $q=-3$ but a similar diagram can also be drawn for $q=+3$ case.

In this case the dimension- 4 operator $\xi v_{R_{a}} v_{R_{3}}$ connecting intermediate scalar $\xi$ to right handed neutrinos $v_{R_{a}} v_{R_{3}}$ is allowed by the symmetries. This operator leads to decay of $\xi$ to right handed neutrinos as shown in Figure $5 \mathrm{~b}$. As can be seen from Figure $5 b$, this decay is again allowed by the residual $\mathcal{Z}_{3}$ symmetry. Since $\xi$ is a dark sector field, its decay ultimately implies

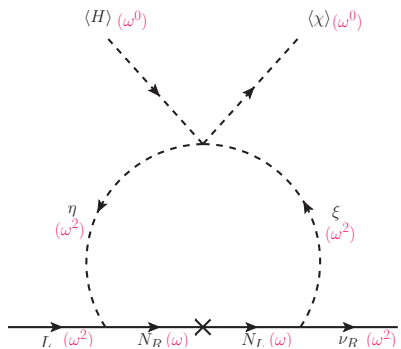

(a) Neutrino mass diagram for $q=-2$ case.

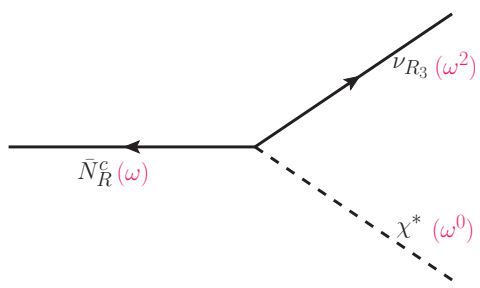

(b) The decay diagram of dark sector particle.

Figure 4: The $q=-2$ case, raditative neutrino mass generation diagram along with residual $\mathcal{Z}_{3}$ charges. Analogous diagram can be drawn for $q=+2$ case, following Figure $2 \mathrm{~b}$. Shown also, the decay diagram of dark sector particle, ultimately leading to the decay of potential dark matter candidate.

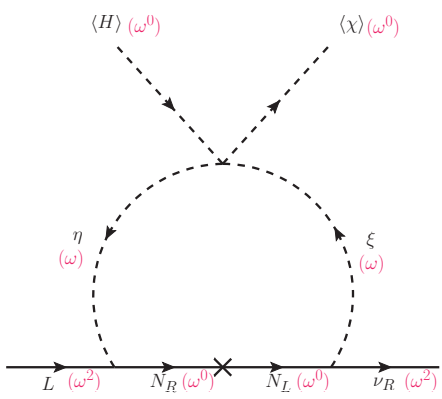

(a) Neutrino mass diagram for $q=-3$ case.

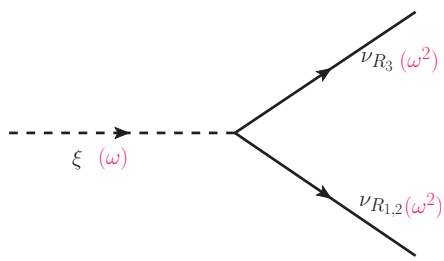

(b) The decay diagram of dark sector particle.

Figure 5: The $q=-3$ case for raditative neutrino mass generation and decay channel of dark sector particles. Analogous diagram can be drawn for $q=+3$ case. 
decay of any potential dark matter candidate. Thus, the dark matter stability is again explicitly lost.

4.0.4. $q= \pm 4$ case

For the case of $q=-4$, the neutrino mass diagram is as shown in Figure 6a. A similar diagram can also be drawn for the $q=$ +4 case as well.

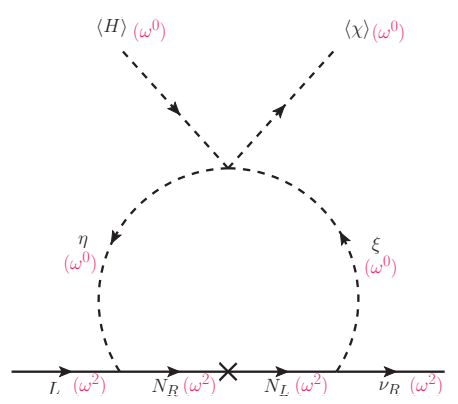

(a) Neutrino mass diagram for $q=-4$ case.

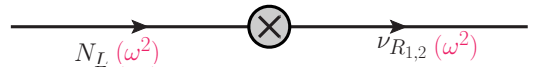

(b) The mixing diagram of dark sector particle.

Figure 6: The $q=-4$ case for raditative neutrino mass generation and mixing diagram of dark sector particle. Analogous diagram can be drawn for $q=+4$ case.

In this case, there is a direct coupling $\bar{N}_{L} v_{R_{a}}$ between the intermediate field $N_{L}$ and the right handed neutrinos $v_{R_{a}}$ as shown in Figure 6b. This coupling leads to mixing between the two fields which is allowed by the residual $\mathcal{Z}_{3}$ symmetry. Owing to this mixing, all the dark sector particles, including any potential dark matter candidate, again have a decay channel available to them. Thus, in this case as well, the dark matter stability is explicitly lost.

\subsection{5. $q= \pm 5$ case}

The Feynman diagram leading to the neutrino mass generation for $q=-5$ is shown in Figure 7a. A very similar diagram can also be drawn for the $q=+5$ case with the fields $N_{L}$ and $N_{R}$ switching their roles.

In this case, analogous to the $q= \pm 4$ case, there is a direct coupling $\bar{N}_{R}^{c} v_{R_{3}}$ allowed by the symmetries. This leads to the mixing between these two fields as shown in Figure $7 \mathrm{~b}$. Again this mixing is allowed by the residual $\mathcal{Z}_{3}$ symmetry and it ultimately leads to the decay of any potential dark matter candidate.

\subsubsection{Accidental symmetry: The $q=0$ and $q= \pm 6$ cases}

Finally, we turn to the cases where an additional accidental symmetry of the model leads to dark matter stability. The presence of accidental symmetries as well as stability of particles owing to accidental symmetries is not a new concept. In the Standard Model, the Lepton $U(1)_{L}$ and Baryon number $U(1)_{B}$ appear as accidental symmetries of the model [35]. As we already saw, the combination $U(1)_{B-L}$ and its residual symmetries are intimately connected with the Dirac/Majorana nature of neutrinos. The other combination $U(1)_{B+L}$ is responsible for stability of proton. Its breaking pattern and residual symme-

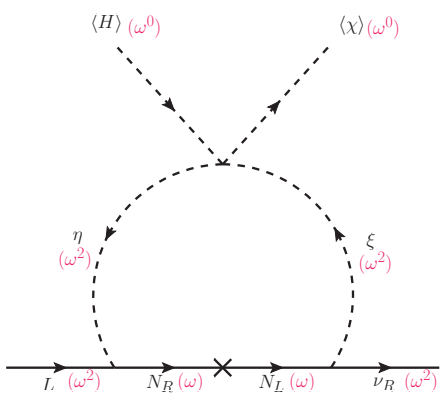

(a) Neutrino mass diagram for $q=-4$ case.

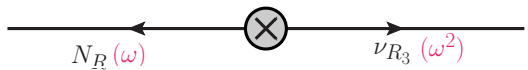

(b) The mixing diagram of dark sector particle.

Figure 7: The $q=-5$ case for raditative neutrino mass generation and mixing diagram of dark sector particle. Analogous diagram can be drawn for $q=+5$ case.

tries are intimately connected with not only stability of proton but also dictate the possible proton decay channels [62, 63, 64]. Just like the accidental $U(1)_{B+L}$ protects proton decay in Standard Model, in many dark matter models an accidental symmetry can protect dark matter decay $[65,24,27]$. A well know example of this is the case of "minimal dark matter" where even after the $\mathrm{SU}(2)_{\mathrm{L}} \otimes \mathrm{U}(1)_{\mathrm{Y}} \rightarrow \mathrm{U}(1)_{\mathrm{em}}$, dark matter remains stable thanks to an accidental symmetry present in the model [65].

In our setup where $U(1)_{B-L} \rightarrow \mathcal{Z}_{3}$, the cases of $q=0$ and $q= \pm 6$ provide such examples. The Feynman diagrams for neutrino mass generation are shown in Figure 8. As can be seen from Figure 8, the intermediate fermions $N_{L}, N_{R}$ do not carry any charge under the residual $\mathcal{Z}_{3}$ symmetry and hence their decays are not protected by it. Furthermore, although the scalar field $\xi$ does carry $\mathcal{Z}_{3}$ charge, still its decay $\xi \rightarrow v_{R}$ is allowed by the residual symmetry. Thus, the dark matter stability in both these cases is not protected by the residual $\mathcal{Z}_{3}$ symmetry. Still the dark matter particle in both these cases is stable thanks to presence of an accidental symmetry in the model.

The presence of an accidental symmetry in both these cases can be seen from the fact that in the unbroken phase the $U(1)_{B-L}$ symmetry allows one to write down decay terms like $\eta^{\dagger} H v_{R_{a}} v_{R_{3}}$ (for $q=0$ ) and $\xi \chi v_{R_{a}}^{c} v_{R_{3}}^{c}$ (for $q= \pm 6$ ). Yet with the particle content of these models, see Table 1, these decay operators cannot be UV completed, clearly indicating presence of an accidental symmetry stabilizing dark matter. This situation is akin to the proton stability in Standard Model where again the gauge symmetries allow non-renormalizable proton decay operators which cannot be UV completed with the particle content of Standard Model [35]. In fact, similar analysis can be carried out for $q<-6$ or $q>6$. In each case it can be shown that the residual $\mathcal{Z}_{3}$ alone cannot stabilize dark matter. In fact it has been shown in [15], arguing at very general grounds that any odd residual $\mathcal{Z}_{n}$ symmetry and in particular $\mathcal{Z}_{3}$ symmetry cannot stabilize dark matter. However, appropriately chosen even $\mathcal{Z}_{n}$ residual symmetries can indeed stabilize dark matter while simultaneously protecting the Dirac nature of neutrinos. In next section, we present one such example. For the most general treatment we refer to [15]. 


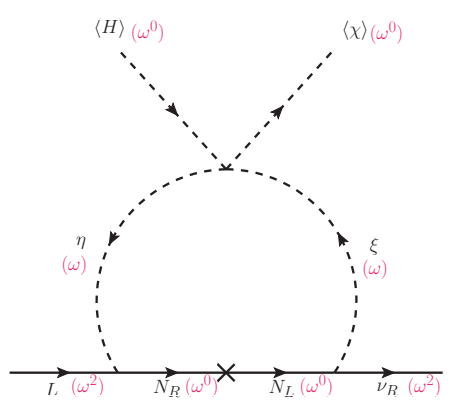

(a) Neutrino mass diagram for $q=0$ case.

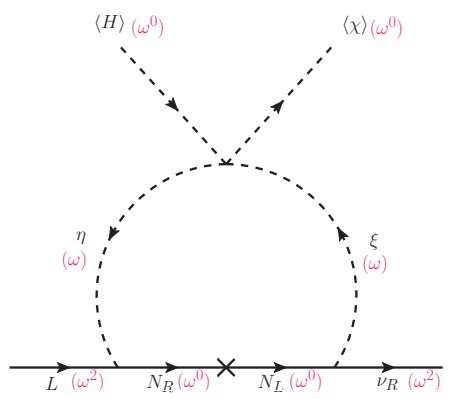

(b) Neutrino mass diagram for $q=-6$ case.

Figure 8: The neutrino mass generation diagrams for both $q=0$ and $q=-6$ cases. In both these cases, the internal fermions do not carry non-trivial $\mathcal{Z}_{3}$ charges. However, one can still have a stable dark matter thanks to the presence of an extra accidental symmetry in the models.

\section{DIRAC NEUTRINOS AND DARK MAT- TER STABILITY FROM A RESIDUAL SYMMETRY: $U\left(\mathbf{1}_{B-L} \rightarrow \mathcal{Z}_{6}\right.$}

We already saw that $U(1)_{B-L} \rightarrow \mathcal{Z}_{3}$ can protect the Dirac nature of neutrinos but $\mathcal{Z}_{3}$ being an odd group, cannot provide dark matter stability. However, if $U(1)_{B-L} \rightarrow \mathcal{Z}_{2 m}, m \in$ $\mathbb{Z}^{+}, m \geq 3$; then one can indeed have dark matter stability as well as protect Dirac nature of neutrinos in a scotogenic like setup [15]. In this section, we show this by explicitly constructing one such example where:

\section{Neutrinos are Dirac in nature.}

II. Neutrino mass is generated at one loop level.

III. The intermediate particles in the loop belong to a "dark sector" with the lightest particle among them being a good candidate for stable dark matter.

We achieve this by $U(1)_{B-L} \rightarrow \mathcal{Z}_{6}$ in the $v_{R}=(-4,-4,5)$ setup with the residual $\mathcal{Z}_{6}$ protecting both Dirac nature of neutrinos and dark matter stability. An alternative model to the one considered here, was also constructed in [15] which the interested reader can consult for more details ${ }^{6}$.

To start with, as discussed before, since the scalar field $\chi \sim$
3 under $U(1)_{B-L}$ symmetry, its vev breaks $U(1)_{B-L} \rightarrow \mathcal{Z}_{3 m}$; $m \in \mathbb{Z}^{+}$. However, exactly which residual $\mathcal{Z}_{3 m}$ symmetry is left unbroken depends on the details of the UV completion and in particular on what is the lowest $U(1)_{B-L}$ charge in the full UV complete theory. In the case, when the Standard Model lepton doublets $L_{i}$ carry the lowest charge i.e., the lowest charge is \pm 1 , then the residual symmetry is $\mathcal{Z}_{3}$. But in case when there is a smaller charge present in the UV complete model, the residual symmetry will be different. For example, if the lowest $U(1)_{B-L}$ charge in the model is $\pm 1 / 2$ then the residual symmetry becomes $\mathcal{Z}_{6}$ while for the lowest charge of $\pm 1 / 3$, the residual symmetry will be $\mathcal{Z}_{9}$, and so on. We will exploit this feature now to break $U(1)_{B-L} \rightarrow \mathcal{Z}_{6}$ by introducing intermediate particles with $\pm 1 / 2$ charges.

For the sake of uniformity and to allow quick comparison, we again take the first topology described in Section 3 and construct a topologically similar model to the ones considered in Section 4. We construct a Standard Model extension where the residual symmetry $\mathcal{Z}_{6}$, stemming from the spontaneous symmetry breaking of $U(1)_{B-L}$, protects the stability of the dark matter candidate and the Dirac nature of neutrinos. As before, we demand that the model is anomaly free. Hence, again the simplest option is to consider that the RH-neutrinos transform as $\left(v_{R_{a}}, v_{R_{3}}\right) \sim(-4,5)$ (with $\left.a=1,2\right)$ under the $U(1)_{B-L}$ symmetry. In order to complete the diagram, as before, we need to introduce three additional scalars, $\chi, \xi$ and $\eta$. The former one is responsible for breaking the $U(1)_{B-L}$ symmetry and the other two will be required to UV complete the model and will belong to the dark sector. A pair of neutral fermions $N_{L}, N_{R}$ are also needed and these along with the scalars $\xi$ and $\eta$ are part of the dark sector with the lightest of them being a good dark matter candidate. In Table 2, we summarize the matter content and the charge assignments of the model. The right column with $\mathcal{Z}_{6}$ label indicates the charge of the particles under the residual $\mathcal{Z}_{6}$ symmetry.

Notice that the intermediate particles carry $U(1)_{B-L}$ charges in units of $1 / 2$. Owing to presence of these particles, the vev of $\chi$ now breaks $U(1)_{B-L} \rightarrow \mathcal{Z}_{6}$. The charges of the fields under the residual $\mathcal{Z}_{6}$ are also listed in Table 2 . Since the lepton doublets $L_{i}$ transform as $\omega^{4}$ under $\mathcal{Z}_{6}$, therefore in accordance with (1) and (2), the neutrinos will be Dirac fermions with their Dirac nature protected by the $\mathcal{Z}_{6}$ symmetry.

The invariant Lagrangian of the theory that describes the neutrino interactions is given by,

$\mathcal{L}_{v}=y^{v} \bar{L} \tilde{\eta} N_{R}+y^{v \prime} \bar{N}_{L} v_{R} \xi+M \bar{N}_{R} N_{L}+$ h.c.

where $\tilde{\eta}=i \tau_{2} \eta^{*}$ and we are omitting flavour indices for convenience. The relevant term, in the scalar potential, to generate the Dirac neutrino mass is given by

$\mathcal{V} \supset \lambda_{D} H^{\dagger} \eta \chi \xi^{*}+$ h.c.,

where $\lambda_{D}$ is an dimensionless quartic coupling.

After spontaneous symmetry breaking two neutrinos acquire a mass through the loop depicted in Figure 9.

${ }^{6}$ For anologous treatment in case of Majorana neutrinos see [16]. 


\begin{tabular}{|c|c|c|c|c|}
\hline & Fields & $S U(2)_{L} \otimes U(1)_{Y}$ & $U(1)_{B-L}$ & $\mathcal{Z}_{6}$ \\
\hline : & $\begin{array}{c}L_{i} \\
v_{R_{a}} \\
v_{R_{3}} \\
N_{L(R)} \\
\end{array}$ & $\begin{array}{c}(\mathbf{2},-1 / 2) \\
(\mathbf{1}, 0) \\
(\mathbf{1}, 0) \\
(\mathbf{1}, 0)\end{array}$ & $\begin{array}{c}1 \\
-4 \\
5 \\
1 / 2 \\
\end{array}$ & $\begin{array}{l}\omega^{4} \\
\omega^{4} \\
\omega^{4} \\
\omega\end{array}$ \\
\hline 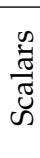 & $\begin{array}{l}H \\
\chi \\
\eta \\
\xi\end{array}$ & $\begin{array}{c}(2,1 / 2) \\
(1,0) \\
(2,1 / 2) \\
(1,0)\end{array}$ & $\begin{array}{c}0 \\
3 \\
3 / 2 \\
9 / 2\end{array}$ & $\begin{array}{c}1 \\
1 \\
\omega^{3} \\
\omega^{3}\end{array}$ \\
\hline
\end{tabular}

Table 2: Matter content and charge assignments of the model. Here $\mathcal{Z}_{6}$ is the residual symmetry with $\omega=e^{2 \pi I / 6} ; \omega^{6}=1$.
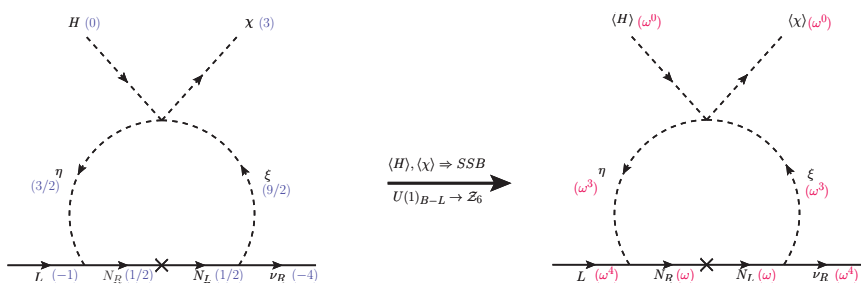

Figure 9: One loop neutrino mass generation diagram highlighting the $U(1)_{B-L} \rightarrow \mathcal{Z}_{6}$ breaking pattern. The $B-L$ charges (left diagram) of the fields are given in blue while the residual $\mathcal{Z}_{6}$ charges (right diagram) are in red. For $\mathcal{Z}_{6}$ symmetry $\omega=e^{2 \pi I / 6} ; \omega^{6}=1$ is the six-th root of unity.

Note that only two RH-neutrinos, $v_{R_{1}}$ and $v_{R_{2}}$, get mass after spontaneous symmetry breaking. The third one $v_{R_{3}}$ remains massless which is consistent with the current experimental data [5]. Furthermore, it is trivial to extend this simple model by adding another vev carrying scalar $\chi_{6} \sim 6$ under $U(1)_{B-L}$ to generate mass for the third neutrino. As mentioned before, since the $U(1)_{B-L}$ charge of $\chi_{6}$ is just an integer multiple of the charge of $\chi$ field, presence of $\chi_{6}$ in the model will not change the $U(1)_{B-L} \rightarrow \mathcal{Z}_{6}$ breaking pattern.

Apart from protecting the Dirac nature of neutrinos, the residual $\mathcal{Z}_{6}$ symmetry also protects the dark matter from decay. To see this, notice that under the $\mathcal{Z}_{6}$ symmetry all intermediate particles carry odd units of the fundamental charge $\omega$, while all the Standard Model particles as well as $v_{R}$ and $\chi$ carry even units of $\omega$ charge. Thus, just like in scotogenic models, here also the intermediate particles split into a separate "dark sector" with the decay of any dark sector particle to only Standard Model particles or to $v_{R}$ and $\chi$, forbidden by the residual $\mathcal{Z}_{6}$ symmetry. Therefore, the lightest of the dark sector particles becomes a good candidate for dark matter with its stability protected by the residual $\mathcal{Z}_{6}$ symmetry. An alternative model employing similar mechanism is also discussed in [15] which can be consulted for more details. For a similar treatment for Majorana neutrinos, we refer to [16].

\section{CONCLUSIONS}

We have discussed the importance of residual symmetries for models that try to make a connection between dark matter stability and the Dirac nature of neutrinos. We showed that in absence of any other conserved symmetry beyond Standard Model symmetries, the Dirac/Majorana nature of neutrinos is dictated by the residual $\mathcal{Z}_{n}$ symmetry left unbroken after $U(1)_{B-L}$ breaking. We then discussed the possible one loop realization of the models that can be constructed where Dirac neutrino mass has a dynamical origin at the 1-loop level. These models employ the chiral solutions to $U(1)_{B-L}$ anomaly free solution. Therefore, the tree level dimension-4 Yukawa term between left and right neutrinos is automatically forbidden by the $U(1)_{B-L}$. The small Dirac mass for neutrinos is then generated through one loop realizations of the dimension- 5 operator $\bar{L} \tilde{H} \chi v_{R}$.

For such one loop models, we also discussed how the same residual $\mathcal{Z}_{n}$ symmetry protecting the Dirac nature of neutrinos, can also protect the dark matter stability by employing a scotogenic like mechanism. We showed that in such scenarios, not all $\mathcal{Z}_{n}$ subgroups can protect the dark matter stability. To this end, we gave several examples where the dark matter stability is lost as it is unprotected by the residual $\mathcal{Z}_{n}$ subgroup. For completeness we also discussed scenarios where a new accidental symmetry might be present in the model, thus protecting the dark matter stability. Finally, we constructed an explicit model with $U(1)_{B-L} \rightarrow \mathcal{Z}_{6}$ breaking pattern, where the Dirac nature of neutrinos and dark matter stability are indeed simultaneously protected by the residual $\mathcal{Z}_{6}$ symmetry.

\section{ACKNOWLEDGMENTS}

We thank Ricardo Cepedello and Salvador Centelles Chuliá for many useful discussions. RS is supported by the Spanish grants SEV-2014-0398 and FPA2017-85216-P (AEI/FEDER, UE), PROMETEO/2018/165 (Generalitat Valenciana) and the Spanish Red Consolider MultiDark FPA2017-90566-REDC. The work of C.B. was supported by the Collaborative Research Center SFB1258. EP is supported by DGAPA-PAPIIT IN107118. RS will like to also thank "El Jefe" for sponsoring his Formula One GP Mexico tickets.

\section{References}

[1] Planck Collaboration, N. Aghanim et al., "Planck 2018 results. VI. Cosmological parameters," arXiv:1807.06209 [astro-ph.CO].

[2] MINOS Collaboration, L. H. Whitehead, "Neutrino Oscillations with MINOS and MINOS+," Nucl. Phys. B908 (2016) 130-150, arXiv: 1601.05233 [hep-ex].

[3] KamLAND Collaboration, M. P. Decowski, "KamLAND's precision neutrino oscillation measurements," Nucl. Phys. B908 (2016) 52-61.

[4] T2K Collaboration, K. Abe et al., "Combined Analysis 
of Neutrino and Antineutrino Oscillations at T2K," Phys. Rev. Lett. 118 no. 15, (2017) 151801, arXiv:1701.00432 [hep-ex].

[5] P. F. de Salas, D. V. Forero, C. A. Ternes, M. Tortola, and J. W. F. Valle, "Status of neutrino oscillations 2018: $3 \sigma$ hint for normal mass ordering and improved CP sensitivity," Phys. Lett. B782 (2018) 633-640, arXiv : 1708.01186 [hep-ph].

[6] P. Minkowski, " $\mu \rightarrow e \gamma$ at a Rate of One Out of $10^{9}$ Muon Decays?," Phys. Lett. 67B (1977) 421-428.

[7] T. Yanagida, "Horizontal symmetry and masses of neutrinos," Workshop on the baryon number of the Universe and unified theories, O. Sawada and A. Sugamoto, eds. (1979) 95.

[8] R. N. Mohapatra and G. Senjanovic, "Neutrino mass and spontaneous parity violation," Phys. Rev. Lett. 44 (1980) 912.

[9] J. Schechter and J. Valle, "Neutrino masses in $\operatorname{SU}(2) \times$ U(1) theories," Phys. Rev. D22 (1980) 2227.

[10] J. Schechter and J. W. F. Valle, "Neutrino Decay and Spontaneous Violation of Lepton Number," Phys. Rev. D25 (1982) 774.

[11] R. Foot, H. Lew, X. G. He, and G. C. Joshi, "Seesaw Neutrino Masses Induced by a Triplet of Leptons," Z. Phys. C44 (1989) 441.

[12] E. Ma, "Pathways to naturally small neutrino masses," Phys. Rev. Lett. 81 (1998) 1171-1174, arXiv:hep-ph/9805219 [hep-ph].

[13] S. Centelles Chuliá, R. Srivastava, and J. W. F. Valle, "Seesaw roadmap to neutrino mass and dark matter," Phys. Lett. B781 (2018) 122-128, arXiv: 1802.05722 [hep-ph].

[14] E. Ma, "Verifiable radiative seesaw mechanism of neutrino mass and dark matter," Phys. Rev. D73 (2006) 077301, arXiv:hep-ph/0601225 [hep-ph].

[15] C. Bonilla, S. Centelles-Chulia, R. Cepedello, E. Peinado, and R. Srivastava, "Dark matter stability and Dirac neutrinos using only Standard Model symmetries," arXiv:1812.01599 [hep-ph].

[16] S. Centelles Chulia, R. Cepedello, E. Peinado, and R. Srivastava, "Scotogenic Dark Symmetry as a residual subgroup of Standard Model Symmetries," arXiv:1901.06402 [hep-ph].

[17] M. Kadastik, K. Kannike, and M. Raidal, "Matter parity as the origin of scalar Dark Matter," Phys. Rev. D81 (2010) 015002, arXiv:0903.2475 [hep-ph].

[18] M. Kadastik, K. Kannike, and M. Raidal, "Dark Matter as the signal of Grand Unification," Phys. Rev. D80 (2009) 085020, arXiv:0907.1894 [hep-ph]. [Erratum: Phys. Rev.D81,029903(2010)].

[19] E. Ma, "Derivation of Dark Matter Parity from Lepton Parity," Phys. Rev. Lett. 115 no. 1, (2015) 011801, arXiv: 1502.02200 [hep-ph].

[20] S. Centelles Chuliá, E. Ma, R. Srivastava, and J. W. F. Valle, "Dirac Neutrinos and Dark Matter Stability from Lepton Quarticity," Phys. Lett. B767 (2017) 209-213, arXiv:1606.04543 [hep-ph].

[21] S. Centelles Chuliá, R. Srivastava, and J. W. F. Valle, "CP violation from flavor symmetry in a lepton quarticity dark matter model," Phys. Lett. B761 (2016) 431-436, arXiv:1606.06904 [hep-ph].

[22] W. Wang and Z.-L. Han, "Naturally Small Dirac Neutrino Mass with Intermediate $S U(2)_{L}$ Multiplet Fields," arXiv: 1611.03240 [hep-ph]. [JHEP04,166(2017)].
[23] S. Centelles Chuliá, R. Srivastava, and J. W. F. Valle, "Generalized Bottom-Tau unification, neutrino oscillations and dark matter: predictions from a lepton quarticity flavor approach," Phys. Lett. B773 (2017) 26-33, arXiv: 1706.00210 [hep-ph].

[24] W. Wang, R. Wang, Z.-L. Han, and J.-Z. Han, "The $B-L$ Scotogenic Models for Dirac Neutrino Masses," Eur. Phys. J. C77 no. 12, (2017) 889, arXiv: 1705.00414 [hep-ph].

[25] Z.-L. Han and W. Wang, " $Z$ ' Portal Dark Matter in $B-L$ Scotogenic Dirac Model," Eur. Phys. J. C78 no. 10, (2018) 839, arXiv:1805.02025 [hep-ph].

[26] G. Lazarides, M. Reig, Q. Shafi, R. Srivastava, and J. W. F. Valle, "Spontaneous Breaking of Lepton Number and Cosmological Domain Wall Problem," arXiv:1806.11198 [hep-ph].

[27] J. Calle, D. Restrepo, C. E. Yaguna, and O. Zapata, "Minimal radiative Dirac neutrino mass models," arXiv:1812.05523 [hep-ph].

[28] E. Ma, "Scotogenic $U(1)_{\chi}$ Dirac Neutrinos," arXiv:1901.09091 [hep-ph].

[29] S. K. Kang, O. Popov, R. Srivastava, J. W. F. Valle, and C. A. Vaquera-Araujo, "Scotogenic dark matter stability from gauged matter parity," arXiv:1902.05966 [hep-ph].

[30] J. C. Montero and V. Pleitez, "Gauging U(1) symmetries and the number of right-handed neutrinos," Phys. Lett. B675 (2009) 64-68, arXiv : 0706.0473 [hep-ph] .

[31] E. Ma and R. Srivastava, "Dirac or inverse seesaw neutrino masses with $B-L$ gauge symmetry and $S_{3}$ flavor symmetry," Phys. Lett. B741 (2015) 217-222, arXiv:1411.5042 [hep-ph].

[32] E. Ma, N. Pollard, R. Srivastava, and M. Zakeri, "Gauge B - L Model with Residual $Z_{3}$ Symmetry," Phys. Lett. B750 (2015) 135-138, arXiv: 1507.03943 [hep-ph].

[33] E. Ma and R. Srivastava, "Dirac or inverse seesaw neutrino masses from gauged B-L symmetry," Mod. Phys. Lett. A30 no. 26, (2015) 1530020, arXiv: 1504.00111 [hep-ph].

[34] M. Hirsch, R. Srivastava, and J. W. F. Valle, "Can one ever prove that neutrinos are Dirac particles?," Phys. Lett. B781 (2018) 302-305, arXiv: 1711.06181 [hep-ph].

[35] S. Weinberg, "Varieties of Baryon and Lepton Nonconservation," Phys. Rev. D22 (1980) 1694.

[36] J. W. F. Valle and C. A. Vaquera-Araujo, “Dynamical seesaw mechanism for Dirac neutrinos," Phys. Lett. B755 (2016) 363-366, arXiv: 1601.05237 [hep-ph].

[37] M. Reig, J. W. F. Valle, and C. A. Vaquera-Araujo, “Realistic $\mathrm{SU}(3)_{\mathrm{c}} \otimes \mathrm{SU}(3)_{\mathrm{L}} \otimes \mathrm{U}(1)_{\mathrm{X}}$ model with a type II Dirac neutrino seesaw mechanism," Phys. Rev. D94 no. 3, (2016) 033012, arXiv:1606.08499 [hep-ph].

[38] S. Centelles Chuliá, "Dirac neutrinos, dark matter stability and flavour predictions from Lepton Quarticity," in 7th International Pontecorvo Neutrino Physics School Prague, Czech Republic, August 20-September 1, 2017. 2017. arXiv:1711.10719 [hep-ph].

[39] D. Borah and A. Dasgupta, "Naturally Light Dirac Neutrino in Left-Right Symmetric Model," JCAP 1706 no. 06, (2017) 003, arXiv:1702.02877 [hep-ph].

[40] C. Bonilla, J. M. Lamprea, E. Peinado, and J. W. F. Valle, "Flavour-symmetric type-II Dirac neutrino seesaw mechanism," Phys. Lett. B779 (2018) 257-261, arXiv: 1710.06498 [hep-ph].

[41] R. Srivastava, C. A. Ternes, M. Tórtola, and J. W. F. Valle, "Testing a lepton quarticity flavor theory of neutrino os- 
cillations with the DUNE experiment," Phys. Lett. B778 (2018) 459-463, arXiv: 1711.10318 [hep-ph].

[42] D. Borah and B. Karmakar, " $A_{4}$ flavour model for Dirac neutrinos: Type I and inverse seesaw," Phys. Lett. B780 (2018) 461-470, arXiv: 1712.06407 [hep-ph].

[43] D. Borah and B. Karmakar, "Linear seesaw for Dirac neutrinos with $A_{4}$ flavour symmetry," Phys. Lett. B789 (2019) 59-70, arXiv:1806.10685 [hep-ph].

[44] Y. Farzan and E. Ma, "Dirac neutrino mass generation from dark matter," Phys. Rev. D86 (2012) 033007, arXiv:1204.4890 [hep-ph].

[45] H. Okada, "Two loop Induced Dirac Neutrino Model and Dark Matters with Global $U(1)^{\prime}$ Symmetry," arXiv:1404.0280 [hep-ph].

[46] C. Bonilla, E. Ma, E. Peinado, and J. W. F. Valle, "Two-loop Dirac neutrino mass and WIMP dark matter," Phys. Lett. B762 (2016) 214-218, arXiv: 1607.03931 [hep-ph].

[47] E. Ma and U. Sarkar, "Radiative Left-Right Dirac Neutrino Mass," Phys. Lett. B776 (2018) 54-57, arXiv: 1707.07698 [hep-ph].

[48] J. C. Helo, M. Hirsch, and T. Ota, "Proton decay and light sterile neutrinos," JHEP 06 (2018) 047, arXiv: 1803.00035 [hep-ph].

[49] M. Reig, D. Restrepo, J. W. F. Valle, and O. Zapata, "Bound-state dark matter and Dirac neutrino masses," Phys. Rev. D97 no. 11, (2018) 115032, arXiv:1803.08528 [hep-ph].

[50] S. K. Kang and O. Popov, "Radiative neutrino mass via fermion kinetic mixing," Phys. Rev. D98 no. 11, (2018) 115025, arXiv: 1807.07988 [hep-ph].

[51] C. D. R. Carvajal and O. Zapata, "One-loop Dirac neutrino mass and mixed axion/WIMP dark matter," arXiv: 1812.06364 [hep-ph].

[52] P. D. Bolton, F. F. Deppisch, C. Hati, S. Patra, and U. Sarkar, "An alternative formulation of left-right symmetry with $B-L$ conservation and purely Dirac neutrinos," arXiv:1902.05802 [hep-ph].

[53] S. Saad, "Simplest Radiative Dirac Neutrino Mass Models," arXiv:1902.07259 [hep-ph].

[54] A. Aranda, C. Bonilla, S. Morisi, E. Peinado, and J. W. F. Valle, "Dirac neutrinos from flavor symmetry," Phys. Rev.
D89 no. 3, (2014) 033001, arXiv:1307.3553 [hep-ph].

[55] J. Heeck and W. Rodejohann, "Neutrinoless Quadruple Beta Decay," EPL 103 no. 3, (2013) 32001, arXiv:1306.0580 [hep-ph].

[56] G. Abbas, S. Gupta, G. Rajasekaran, and R. Srivastava, "High Scale Mixing Unification for Dirac Neutrinos," Phys. Rev. D91 no. 11, (2015) 111301, arXiv:1312.7384 [hep-ph].

[57] G. Abbas, M. Z. Abyaneh, and R. Srivastava, "Precise predictions for Dirac neutrino mixing," Phys. Rev. D95 no. 7, (2017) 075005, arXiv: 1609.03886 [hep-ph].

[58] E. Ma and O. Popov, "Pathways to Naturally Small Dirac Neutrino Masses," Phys. Lett. B764 (2017) 142-144, arXiv:1609.02538 [hep-ph].

[59] C.-Y. Yao and G.-J. Ding, "Systematic analysis of Dirac neutrino masses from a dimension five operator," Phys. Rev. D97 no. 9, (2018) 095042, arXiv:1802.05231 [hep-ph].

[60] C.-Y. Yao and G.-J. Ding, "Systematic Study of One-Loop Dirac Neutrino Masses and Viable Dark Matter Candidates," Phys. Rev. D96 no. 9, (2017) 095004, arXiv:1707.09786 [hep-ph]. [Erratum: Phys. Rev.D98,no.3,039901(2018)].

[61] S. Centelles Chuliá, R. Srivastava, and J. W. F. Valle, "Seesaw Dirac neutrino mass through dimensionsix operators," Phys. Rev. D98 no. 3, (2018) 035009, arXiv:1804.03181 [hep-ph].

[62] P. Nath and P. Fileviez Perez, "Proton stability in grand unified theories, in strings and in branes," Phys. Rept. 441 (2007) 191-317, arXiv:hep-ph/0601023 [hep-ph] .

[63] R. M. Fonseca, M. Hirsch, and R. Srivastava, " $\Delta L=3$ processes: Proton decay and the LHC," Phys. Rev. D97 no. 7, (2018) 075026, arXiv:1802.04814 [hep-ph].

[64] M. Reig and R. Srivastava, "Spontaneous proton decay and the origin of Peccei-Quinn symmetry," Phys. Lett. B790 (2019) 134-139, arXiv: 1809.02093 [hep-ph].

[65] M. Cirelli, N. Fornengo, and A. Strumia, "Minimal dark matter," Nucl. Phys. B753 (2006) 178-194, arXiv:hep-ph/0512090 [hep-ph]. 